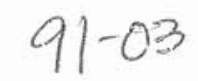

\title{
The Electron Absorption Signature of 1989N1
}

\author{
R. S. SELESNICK AND E. C. STONE \\ California Institute of Technology, Pasadena
}

\begin{abstract}
A deep minimum in the energetic $(21 \mathrm{MeV})$ electron intensity at Neptune is associated with absorption by the satellite $1989 \mathrm{~N} 1$. The absorption signature is modeled by assuming that the electron losses are replenished by diffusion due to violation of the third adiabatic invariant. It is predominantly a steady state feature, or macrosignature, for which approximate solutions of the diffusion equation lead to an estimate of the radial diffusion coefficient, $D_{L L} \approx 2 \times 10^{-8} \mathrm{~s}^{-1}$ at $L=5$. The model suggests a strong $L$ dependence for $D_{L L}\left(\sim L^{7}\right)$, but an outward displacement of the macrosignature by $\sim 0.3$ unit in $L$ from the location predicted by the model, and differences in the observed and model energy spectra, may indicate that processes such as pitch angle and energy diffusion need to be included for an accurate assessment of the transport coefficients. An approximate solution is also found for the time-dependent, two-dimensional diffusion equation including particle drifts and satellite absorption. It shows that the transient microsignatures of the satellite are largely dispersed by drifts and filled in by diffusion. However, the deep intensity minimum appears to include microsignatures near each of the two satellite minimum orbital $L$ shell locations, and these are expected from the diffusion model. At the time of the observations the satellite had recently encountered the inner location, and its microsignature was not yet significantly affected by diffusion. The observed microsignatures are closer together than the minimum orbital $L$ shell values based on the magnetic field model and, although they are nearly consistent with absorption by 1989 N1 alone, the possibility that one was formed by other orbital material cannot presently be ruled out.
\end{abstract}

\section{INTRODUCTION}

Satellites orbiting within the intense radiation belts of the outer planets can absorb significant fluxes of charged particles and produce observable signatures in the data returned by particle detectors, especially at high particle energies [e.g., Thomsen, 1979 and references therein; Van Allen et al., 1980; Simpson et al, 1980; Stone et al., 1986, 1989]. These signatures can be useful in studying the properties and orbits of the satellites [e.g., Chenette and Stone, 1983; Cuzzi and Burns, 1988], the geometry of the planetary magnetic field [e.g., Chenette and Davis, 1982], and particle transport in the radiation belts [e.g., Thomsen et al., $1977 a, b ; \mathrm{Hood}, 1989 a, b]$. The localized nature of the particle losses can lead to particularly strong constraints on particle diffusion models. Such modeling of Neptune's trapped electrons, with absorption by the satellite $1989 \mathrm{~N} 1$, is the focus of this study.

An overview of the energetic electron flux observed by the Voyager 2 cosmic ray (CRS) experiment in Neptune's magnetosphere is shown in Figure 1. Counting rates versus time from two detectors (D1 responding to electrons with kinetic energy $E \gtrsim 1 \mathrm{MeV}, \mathrm{D} 2$ with $E \gtrsim 2.5 \mathrm{MeV}$ ) are plotted. Detailed descriptions of these results are given by Stone et al. [1989]. The last deep minima in the counting rates, near 0510 SCET (spacecraft event time), are interpreted as absorption signatures of $1989 \mathrm{~N} 1$, which orbits at a distance of $4.75 R_{N}$ (Neptune radii, 1 $R_{N}=24765 \mathrm{~km}$ ).

Section 2 describes in detail the CRS electron observations and spacecraft trajectory near the $1989 \mathrm{~N} 1$ signatures. Section 3 provides, with application to $1989 \mathrm{~N} 1$, a prescription for modeling satellite absorption signatures as observed by charged particle detectors and deriving radiation belt diffusion coefficients. Section 4 is a discussion of the results and their implications for particle diffusion and Neptune's magnetic field.

Copyright 1991 by the American Geophysical Union.

Paper number $91 \mathrm{JA} 01648$. $0148-0227 / 91 / 91 J A-01648 \$ 05.00$

\section{DATA AND ENCOUNTER GEOMETRY}

\subsection{CRS Observations}

The two CRS counting rates from Figure 1 are shown in closeup, near the $1989 \mathrm{~N} 1$ signatures, in Figure 2. The data points were taken every $96 \mathrm{~s}$. In addition, high time resolution (6s) data from D1 are shown in the inset for times after 0508 SCET (the time of an instrumental mode change which shifted the 6-s readout from another CRS detector). The data in Figure 2 have been corrected for the constant background level of 25 counts $\mathrm{s}^{-1}$ due to penetrating cosmic rays, seen near the beginning and end of the Figure 1 data, and for electronic dead time $(20 \mu \mathrm{s})$. The background subtraction significantly affects only the D2 counting rate near the absorption signature minimum. The dead time correction significantly increases the D1 counting rate over the uncorrected values in Figure 1 only near the maximum flux levels. With these corrections the data are linear with respect to the electron intensity.

The counting rates in Figures 1 and 2 are from the D1 and D2 detectors of the CRS electron telescope [Stone et al., 1977]. No coincidence or anticoincidence requirements were made on the events recorded by these detectors while inside Neptune's magnetosphere. Therefore electron energy thresholds and directional acceptance cones are not sharply defined. Instead, detector response functions, $R(E, \theta)$, are defined as a function of the electron kinetic energy $E$ and the angle $\theta$ from the detector symmetry axis:

$$
r=\int_{0}^{\infty} \int_{0}^{2 \pi} \int_{0}^{\pi} R(E, \theta) j(E, \alpha(\theta, \phi)) \sin \theta d \theta d \phi d E
$$

where $r$ is the linear counting rate, $j$ is the electron differential directional intensity, $\alpha$ is the electron pitch angle, and $\phi$ is the azimuthal angle relative to the detector symmetry axis. The response functions have been established by laboratory calibration [Selesnick and Stone, 1991] and are shown in Figure 3. They are seen to vary smoothly with electron energy and with the angle of incidence relative to the detector axis. The contour plots in 


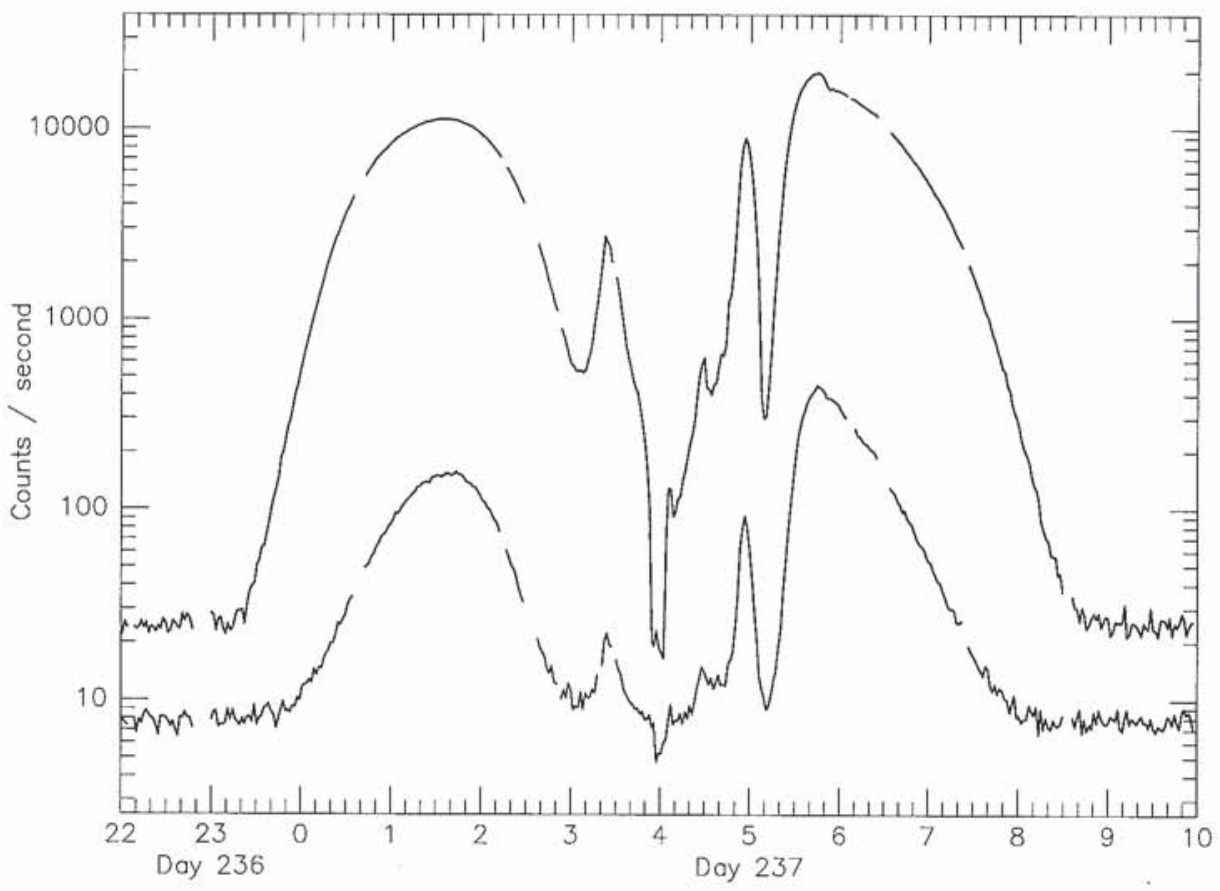

Fig. 1. Counting rates versus spacecraft event time (SCET) from two CRS particle detectors (D1 above, D2 $\times 0.3$ below) in Neptune's magnetosphere. Between $\sim 0130$ and $\sim 0830$ SCET they are responding primarily to trapped electrons. The signatures of $1989 \mathrm{~N} 1$ are the deep minima near 0510 SCET.

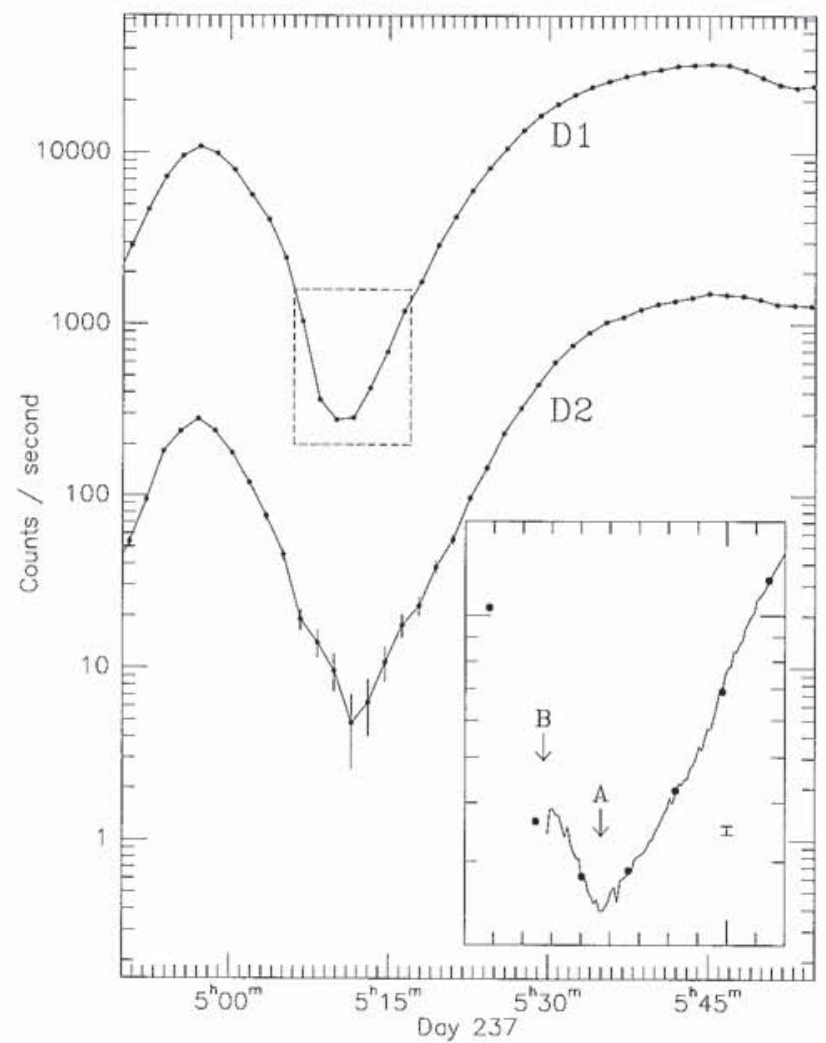

Fig. 2. A close-up view of the counting rates from Figure 1. The individual data points represent 6-s averages spaced by $96 \mathrm{~s}$. The inset, corresponding to the region inside the dashed box, shows additional high time resolution data (6-s averages spaced by $6 \mathrm{~s}$ ) for the D1 detector when it became available after 0508 SCET, along with the low time resolution data points. The labels A and B indicate the absolute D1 counting rate minimum and another local counting rate minimum, respectively. A statistical error bar is shown to the right in the inset at 350 counts s-1 and applies to both the high and low time resolution data.
Figure 3 would be peaked at $\theta=45^{\circ}$ for a bare detector. The D1 response is weighted toward the forward direction (small $\theta$ ) because of collimation by the telescope casing. The D2 response is weighted toward large $\theta$ because electrons from the forward direction must penetrate D1. For D1 an energy threshold of $\sim 1$ $\mathrm{MeV}$ is a reasonable approximation to the energy response. For D2, 2.5 MeV is a similar but less precise approximation. The actual calibrated response functions are used in the analysis presented below.

\subsection{Spacecraft and Satellite Trajectories}

The trajectories are most usefully represented in magnetic coordinates [McIlwain, 1966; Roederer, 1970], which we briefly review for application to the I8E1 spherical harmonic model of Neptune's magnetic field [Connerney et al., this issue]. The $L$ shell parameter is defined by

$$
\frac{L^{3} R_{N}^{3} B_{m}}{k_{0}}=F\left(\frac{K^{3}}{B_{m}^{1 / 2} k_{0}}\right)
$$

where $F$ is a function of the bounce motion that is evaluated numerically (with the power series expansion tabulated by McIlwain or Roederer). The mirror point magnetic field $B_{m}$ is related to the first adiabatic invariant $M$ by

$$
M=\frac{p^{2}}{2 m B_{m}}
$$

where $p$ and $m$ are the electron momentum and rest mass, respectively. The second adiabatic invariant is

$$
K=\int_{s_{1}}^{s_{2}}\left(B_{m}-B\right)^{1 / 2} d s
$$

where $B$ is the local magnetic field and the integral extends along a field line between the mirror points $s_{1}$ and $s_{2}$. The planetary 


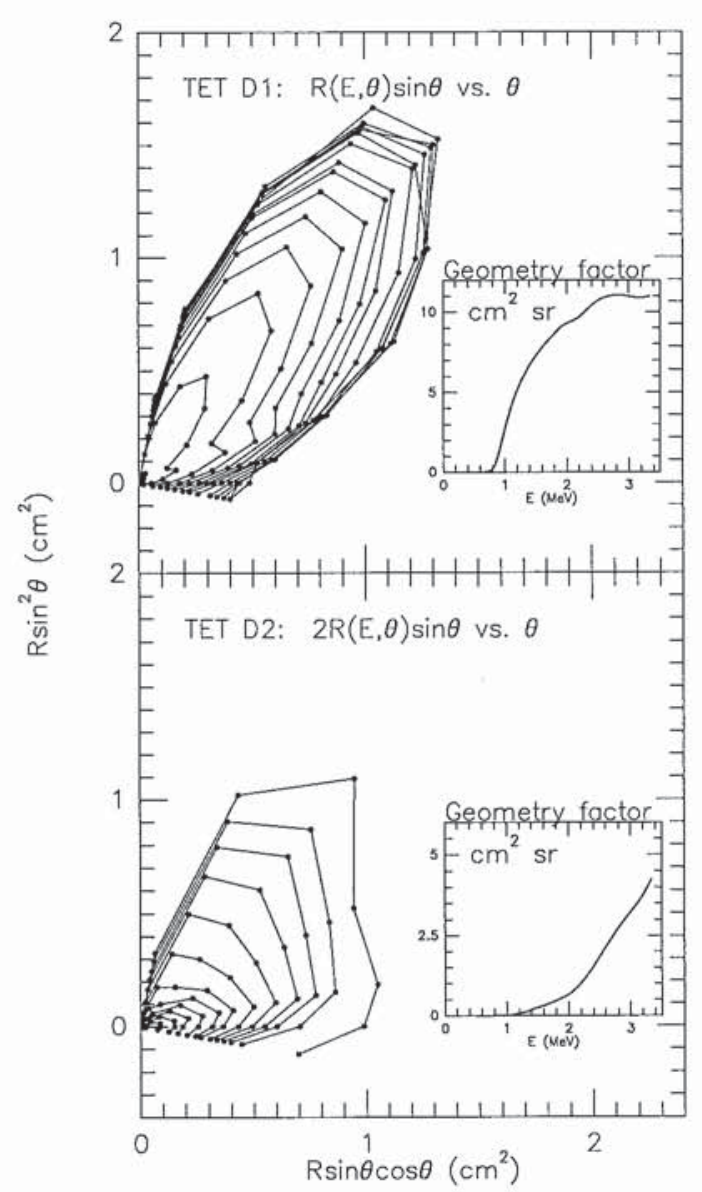

Fig. 3. Contours at fixed energy of the D1 and D2 response functions multiplied by $\sin \theta$ for $\mathrm{D} 1$ and $2 \sin \theta$ for $\mathrm{D} 2$, versus $\theta$ in polar coordinates. The polar angle $\theta$ is from the detector symmetry axis. The contours correspond to electron kinetic energies in $\mathrm{MeV}$, increasing outward, of $0.80,0.99,1.18,1.37,1.57,1.76,1.96,2.16,2.35,2.55,2.75,2.95,3.15$, and 3.35 (the lowest two energies are not visible for D2). The insets show the energy-dependent geometry factors for each detector, which are the response functions integrated over solid angle.

dipole moment, $k_{0}$, must also be specified, and we choose the value of $k_{0}=0.13 \mathrm{G} R_{N}^{3}$, corresponding to the best fit dipole for the Voyager magnetic field data [Ness et al., 1989]. Consistent values of $k_{0}$ must be used in any comparison of derived $L$ values, but the exact numerical value is not significant. The $R$ and $\Lambda$ (invariant latitude) coordinates are defined from $L$ and $B$ according to

$$
\begin{gathered}
R=L \cos ^{2} \Lambda \\
B=\frac{k_{0}}{R^{3} R_{N}^{3}}\left(4-\frac{3 R}{L}\right)^{\frac{1}{2}}
\end{gathered}
$$

These provide a meridional dipolelike representation of the position in the nondipolar magnetic field. The $L$ shell values used below are computed in the I8E1 model for particles with their mirror points at the location of the spacecraft or the satellite. This is a reasonable approximation, as drift shell splitting [Stone, 1963 ] is negligible ( $\lesssim 1 \%$ between the spacecraft and satellite) at the radial positions considered.

A polar plot in $R-\Lambda$ coordinates of the Voyager 2 trajectory through the inner magnetosphere is shown in Figure 4. Also shown are contours of mirror point location for particles of constant $K$ values, and two $L$ shells corresponding to the

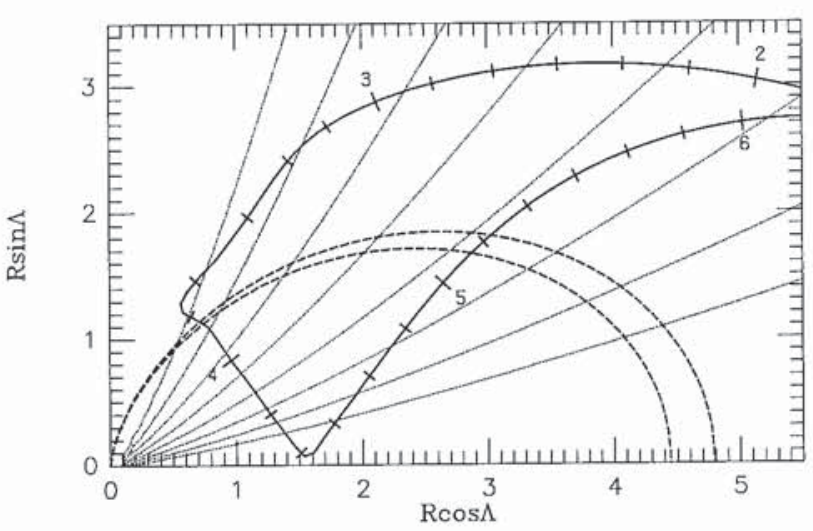

Fig. 4. The Voyager 2 trajectory in magnetic coordinates for the I8E1 field model. The tick marks are at 10 -min intervals and are labeled at the beginning of each hour. The dashed dipole field lines correspond to the two orbital minimum $L$ shells reached by $1989 \mathrm{~N} 1$. The approximately radial contours are the mirror point locations of electrons with constant second adiabatic invariant values ranging from $K=0.025$ to $3.2 \mathrm{G}^{1 / 2} \mathrm{R}_{\mathrm{N}}$ by factors of two.

minimum orbital $L$ values of $1989 \mathrm{~N} 1$. The counting rate minima in Figure 2 at $~ 0510$ SCET are seen to occur between these two $L$ shells and at fairly high invariant latitude. Note that the spacecraft also crossed the minimum $L$ shells of $1989 \mathrm{~N} 1$ at 0352 SCET according to the I8E1 model. However, the spacecraft was moving rapidly through $L$ space at this time, and possible absorption signatures of $1989 \mathrm{~N} 1$ and the other inner moons and rings have yet to be unambiguously identified in the CRS data.

The Voyager 2 and $1989 \mathrm{~N} 1$ trajectories in $L$ versus magnetic longitude are shown in Figure 5. When the absorption signatures were observed ( 0510 SCET), the satellite was near its inner minimum orbital $L$ shell at magnetic longitudes slightly west of the spacecraft. The effects of the satellite on electrons drifting westward would not have been observed at the spacecraft until nearly a full drift period after they occurred. The drift period for $1-\mathrm{MeV}$ electrons is $\sim 2$ hours. The satellite orbital period in magnetic longitude is 39.75 hours, whereas the spacecraft traversed the inner $L$ shell region in only $\sim 2$ hours.

\section{MODELING THE ABSORPTION SIGNATURE}

Satellite absorption of trapped energetic charged particles is a highly localized process, the dimensions of the satellite being generally small compared to the observational scale sizes of the radiation belts. Therefore it is a good approximation to assume that the satellite sweeps up particles only on the drift shell and only at the drift phase (magnetic longitude) that is instantaneously occupied by its center. With this assumption we can define the local particle lifetime for satellite absorption, $\tau_{s}$, by

$$
\tau_{S}^{-1}=\Delta L_{S} \omega_{d} \delta\left(L-L_{S}(t)\right) \delta\left(\phi-\phi_{S}(t)\right)
$$

where $L_{S}(t)$ and $\phi_{S}(t)$ are the $L$ shell and drift phase, $\phi$, respectively, of the satellite orbit at time $t$. The particle bounceaveraged drift frequency is $\omega_{d}$, and the radial width of the satellite is defined as

$$
\Delta L_{s}=\frac{d L_{s}}{d t} \Delta t_{S}
$$

where $\Delta t_{S}$ is the $L$ shell contact time. Under this approximation the dimensions of the satellite enter only into the calculation of $\Delta t_{S}$, which is plotted versus $L$ for $1989 \mathrm{~N} 1$ in Figure 6 . 


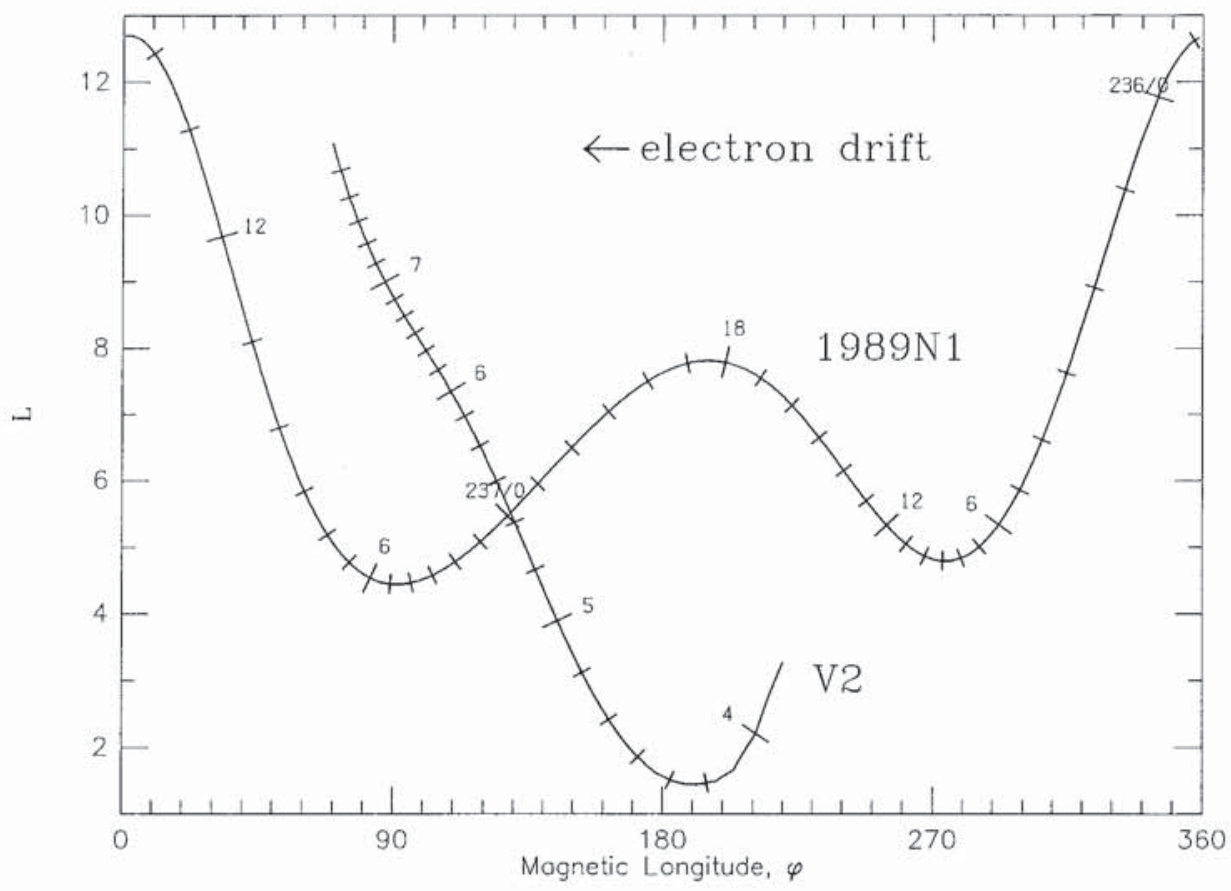

Fig. 5. Voyager 2 and 1989 N1 $L$ versus magnetic longitude. The magnetic longitude is defined relative to the dipole axis of the OTD2 magnetic field model [N. Ness, private communication, 1990] (the OTD2 model is similar to the OTD model of Ness et al., [1989]). The tick marks on the Voyager 2 trajectory are at ten minute intervals and are labeled at the beginning of each hour of day 237. Those on the $1989 \mathrm{~N} 1$ trajectory are at 1-hour intervals and are labeled at the beginning of every sixth hour for days 236 and 237 of 1989.

Substituting (7) into (6) introduces the quantity $\Delta t_{s} \omega_{d}$, which is the azimuthal range absorbed during a given sweeping episode.

The lifetime (6) should strictly contain a factor for the probability of absorption on any drift encounter with the satellite location [Paonessa and Cheng, 1987; Fillius, 1988]. For electrons with the energies of interest, however, it is a good approximation to assume that they are all absorbed (probability $=$
1) [Cooper, 1990] because the drift motion during a half-bounce period is small relative to the satellite radius. Also, $\omega_{d}$ should be computed relative to the satellite, but the orbital period of $1989 \mathrm{~N} 1$ is long enough that its motion can be neglected relative to the electron drift motion in a reference frame rotating with the planet.

The $\phi$ and $t$ dependencies of $\tau_{S}$ require that the phase space density, $f(L, \phi, t)$, also include such dependencies and be

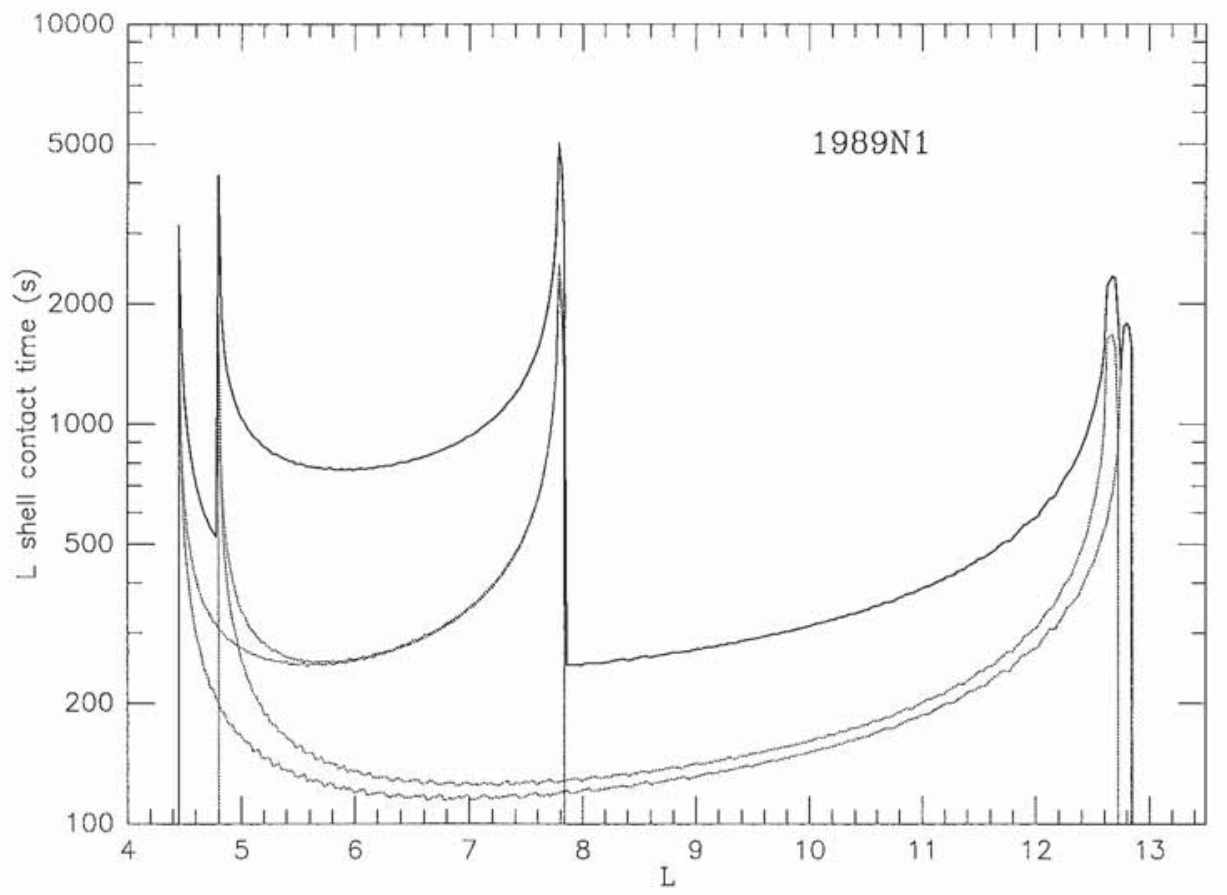

Fig. 6. The $L$ shell contact time in seconds for 1989 N1 versus $L$. The lower curves are for individual encounters with each $L$ during one satellite orbit, of which there are up to four. The upper curve is the orbital total. 
described by a diffusion equation of the form

$$
\frac{\partial f}{\partial t}+\omega_{d} \frac{\partial f}{\partial \phi}=L^{2} \frac{\partial}{\partial L}\left[\frac{D_{L L}}{L^{2}} \frac{\partial f}{\partial L}\right]+D_{\phi \phi} \frac{\partial^{2} f}{\partial \phi^{2}}-\tau_{s}^{-1} f
$$

where $D_{L L}$ and $D_{\phi \phi}$ are radial and azimuthal diffusion coefficients, respectively [Birmingham et al., 1967]. We assume that the diffusion occurs only by violation of the third adiabatic invariant so that the partial derivatives in (8) are taken at constant values of the first two adiabatic invariants, $M$ and $K$.

Useful approximate solutions of the diffusion equation (8) can be obtained from the joint concepts of macrosignatures and microsignatures, introduced by Van Allen et al. [1980] to describe large- and small-scale satellite signatures, respectively. We introduce an average signature and a local signature, which will turn out to be closely related to the macrosignature and microsignature, respectively, and may be concisely defined as follows: The average signature, $f_{A}(L)$ is the average of $f$ over $\phi$ and $t$ during a satellite orbital period $T_{S}$. The local signature, $f_{L}(L, \phi, t)$, is then simply the difference between $f$ and $f_{A}$,

$$
\begin{gathered}
f_{A}(L)=\langle f(L, \phi, t)\rangle_{\phi, t} \\
f_{L}(L, \phi, t)=f-f_{A}
\end{gathered}
$$

\subsection{The Average Signature}

An approximate equation for $f_{A}$ is obtained by replacing $f$ with $f_{A}$ in the last term of the diffusion equation (8), substituting (6) and (7) for $\tau_{s}^{-1}$ and averaging the equation over $\phi$ and $t$. The result is

$$
L^{2} \frac{\partial}{\partial L}\left(\frac{D_{L L}}{L^{2}} \frac{\partial f_{A}}{\partial L}\right)=\left\langle\tau s^{-1}\right\rangle f_{A}
$$

so that $f_{A}$ approximately satisfies a steady state lossy radial diffusion equation. The validity of the approximation will be addressed below. The average lifetime is given by

$$
\left\langle\tau_{s}^{-1}\right\rangle=\sum_{j} \frac{\Delta t_{S j} \omega_{d}}{2 \pi T_{S}}
$$

Here we have introduced the notation that quantities with the subscript $j$ are functions of $L$ and are evaluated at the $j$ th satellite encounter with that $L$ shell during one satellite orbit. The summation extends over all such encounters. If the satellite orbit were not periodic in magnetic coordinates, then the averaging period for $f_{A}$ would be many orbits and the summation would extend over all encounters during that period. For $1989 \mathrm{~N} 1$ a periodic orbit is a good approximation, as the minimum $L$ shell values for successive orbits are nearly equal. The average diffusion equation (10) has been used extensively, although without rigorous derivation, by previous authors [e.g., Thomsen et al., 1977b; Hood, 1989a,b; Selesnick and Stone, 1991]. Various approximations have been adopted for the average lifetime, $\left\langle\tau_{s}^{-1}\right\rangle$, and (11) agrees with our earlier formulation [Selesnick and Stone, 1991] which is closely related to the others.

Adopting boundary conditions in $L$, the average diffusion equation (10) is easily solyed numerically for $f_{A}(L)$ at fixed values of $M$ and $K$ by the relaxation method of Press et al. [1986, chapter 16]. Since the equation is linear, only one iteration of the procedure is required. Sample solutions with absorption by $1989 \mathrm{~N} 1$ are shown in Figure 7 . The drift frequency, $\omega_{d}$, was evaluated with the expression for a dipole magnetic field given by Schulz and Lanzerotti [1974], but using $L$ values from the I8E1

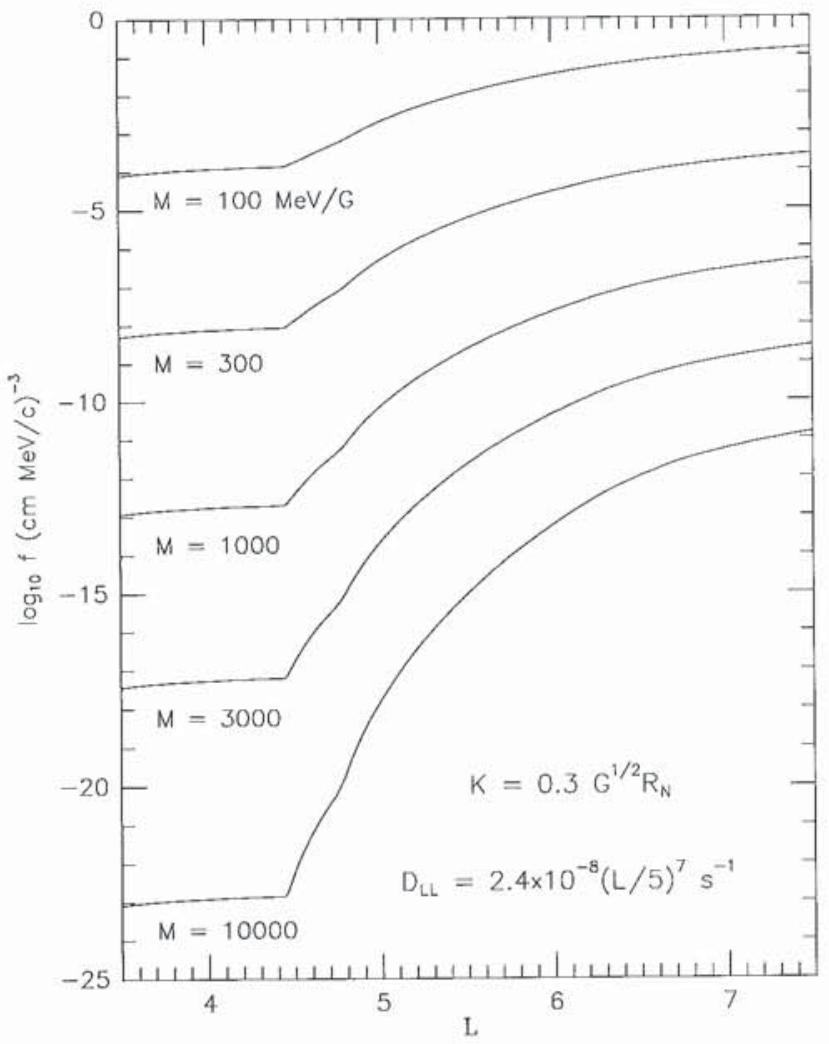

Fig. 7. Solutions of the average diffusion equation (10) versus $L$ for a single value of the second adiabatic invariant, $K$, and various values of the first adiabatic invariant, $M$.

magnetic field model. The outer boundary conditions were chosen at $L=7.5$ from a differential intensity, $j=p^{2} f$, with a power law energy spectrum and power law in the sine of the equatorial pitch angle. The normalization and spectral indices were chosen to give agreement with the data as described below. The inner boundary conditions are $f=0$ at $L=3$, the approximate location of strong absorption by the inner moons and rings. The solutions for $L \gtrsim 3.5$ are insensitive to the inner boundary condition.

The main feature of the solutions in Figure 7 is the large change in the slope of $f_{A}$ at $L=4.45$, the location of the inner minimum orbital $L$ of $1989 \mathrm{~N} 1$ where there is a sharp peak in $\Delta t_{S}$ (Figure 6). A similar peak in $\Delta t_{s}$ occurs at $L=4.79$, the outer orbital minimum $L$, but because of the proximity of the inner peak there is only a small change in the slope of $f_{A}$ at that location. The peaks in $\Delta t_{S}$ at the maximum orbital $L$ values are not significant because the satellite was then at high magnetic latitudes where only particles with small pitch angles can be absorbed. The pitch angles represented in Figure 7 cannot reach these latitudes.

Given solutions for $f_{A}(L)$ at many values of $M$ and $K$, simulations of the CRS counting rates may be constructed for comparison with the observations. This is done by converting from $M$ and $K$ to kinetic energy $E$ and pitch angle $\alpha$ at each $L$ and then numerically evaluating the counting rate integral (1) with $j=p^{2} f_{A}$. Simulations of the D1 and D2 counting rates are shown for three cases in Figure 8. The parameters of Figure $8 a$ are identical with those of Figure 7 . In each case the outer boundary condition is $j(L=7.5)=2.2 \times 10^{5} E^{-5.7} \sin ^{4} \alpha_{0}$ $\left(\mathrm{cm}^{2} \mathrm{~s} \mathrm{srMeV}\right)^{-1}$, where $\alpha_{0}$ is the equatorial pitch angle. The dependence of $j(L=7.5)$ on $E$ is constrained by the relative 

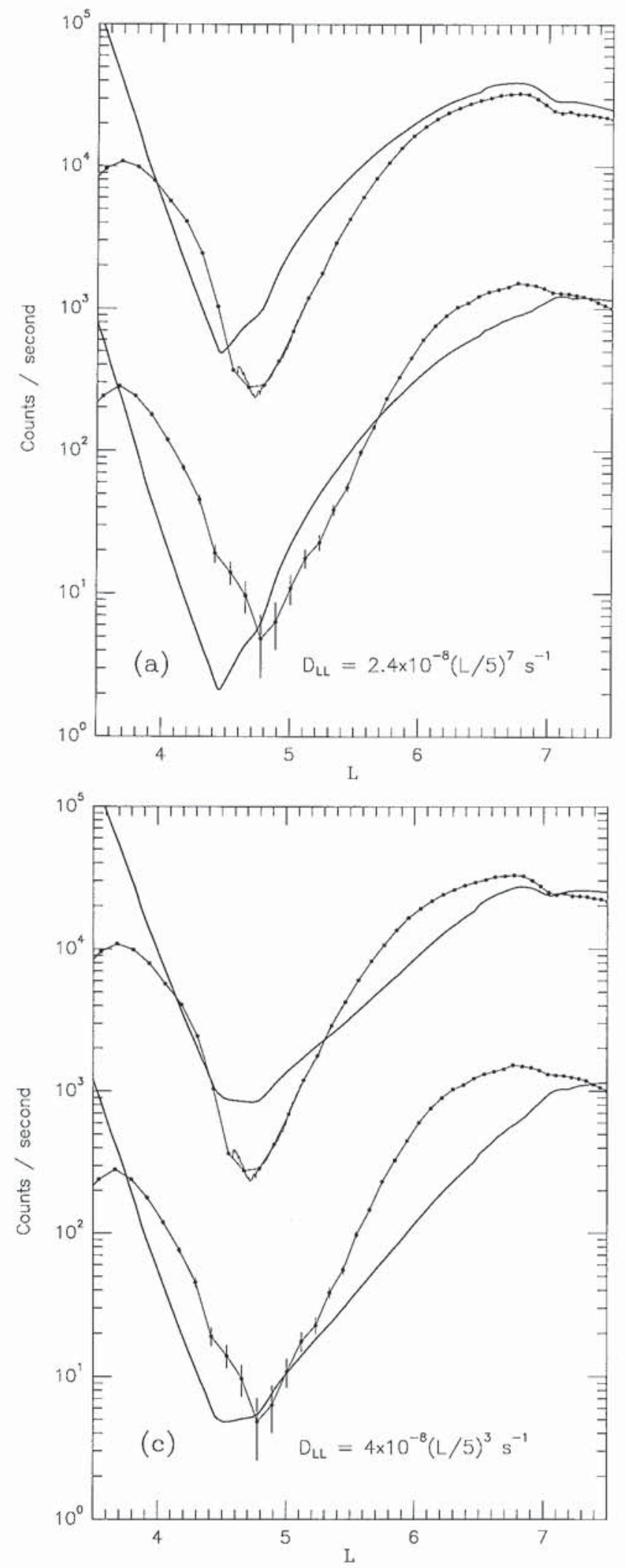

counting rates in D1 and D2 at the outer boundary. The dependence of $j(L=7.5)$ on $\alpha_{0}$ is constrained by the shape of the feature in the D1 rate at $L \approx 7$. The feature is a result of a spacecraft roll maneuver that changed the orientation of the detectors relative to the local magnetic field direction. The feature is less distinct in the D2 rate because that detector has a less directional response function (Figure 3 ). The energy and pitch angle distributions inside $L=7.5$ are determined by the

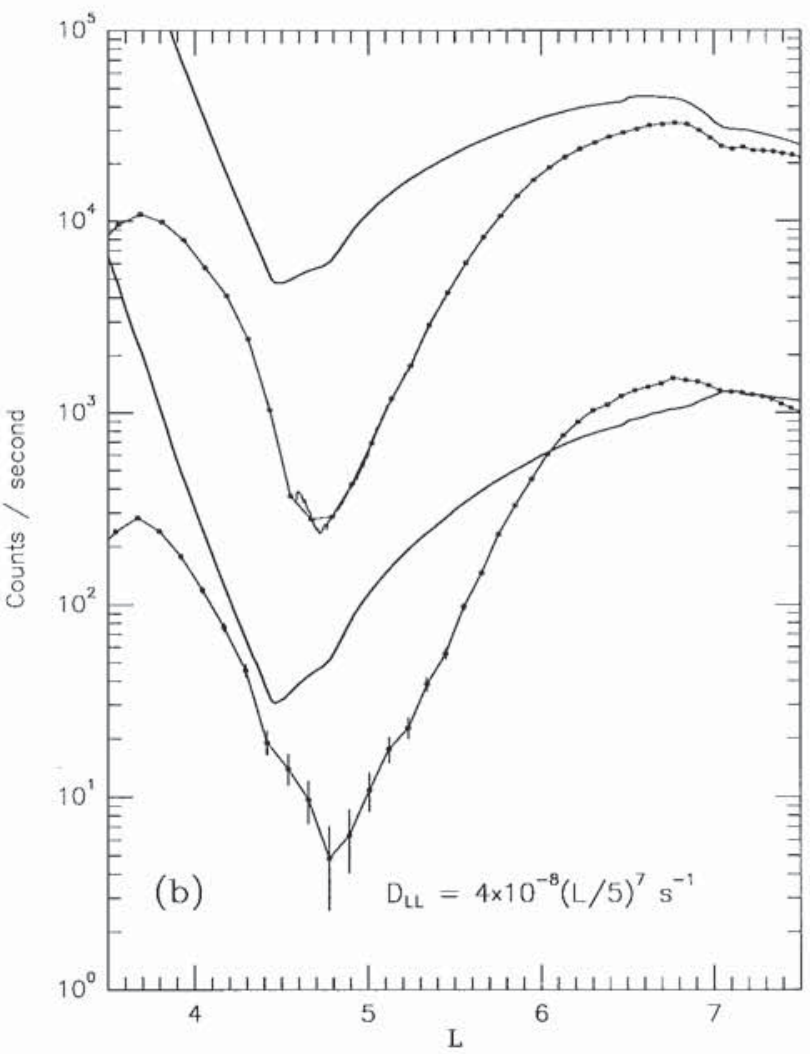

Fig. 8. Simulations of the counting rates (solid curves) in the CRS D1 and D2 detectors based on solutions of the average diffusion equation. The high and low resolution data from Figure 2 are also shown for comparison. Figures $8 a, 8 b$, and $8 c$ differ only in the value of the radial diffusion coefficient as indicated on the figure.

combination of the boundary conditions with the solutions of the diffusion equation. The only difference in the parameters for the three cases is the radial diffusion coefficient, $D_{L L}$, whose value and $L$ dependence is indicated on each plot. Figure $8 a$, which is the closest match between simulations and data, shows that the average signature can be similar in shape and dimensions to the observed signatures. Figures $8 b$ and $8 c$ show the sensitivity of the model to changes in the diffusion coefficient. The signature is 
relatively insensitive to uncertainties in the energy and pitch angle spectral indices used as boundary conditions in the model and determined by comparison with the data. The predicted locations and relative depths of the counting rate minima do not agree exactly with those in the data. We return to these points in section 4 .

\subsection{The Microsignature}

The $\phi$ and $t$ dependencies of the local signature, $f_{L}$, require a solution of the full diffusion equation (8). An approximate analytic solution can be obtained if the $L$ dependence of $\omega_{d}$ is ignored. For simplicity we also replace the radial diffusion term by $D_{L L} \partial^{2} f / \partial L^{2}$ and ignore any $L$ dependence of the diffusion coefficients. These approximations are justified by the smallscale nature of the microsignature. Then a solution for an initial condition $f=f_{0}(L)$ at $t=0$ is

$$
\begin{aligned}
f & =\int d L^{\prime}\left(\frac{f_{0}}{\left(4 \pi D_{L L} t\right)^{1 / 2}} e^{\frac{-\left(L-L^{\prime}\right)^{2}}{4 D_{L L^{\prime}}}}\right. \\
& \left.-\sum_{j} f\left(L^{\prime}, \phi_{S j}, t_{S j}\right) \frac{\Delta t_{S j} \omega_{d}}{4 \pi\left(D_{L L} D_{\phi \phi}\right)^{1 / 2} \delta t_{S j}} e^{\frac{-1}{4 \delta t_{S j}}\left[\frac{\left(L-L^{\prime}\right)^{2}}{D_{L L}}+\frac{\delta \phi_{d j}^{2}}{D_{\phi \phi}}\right]}\right)(12)
\end{aligned}
$$

where

$$
\begin{array}{r}
\delta \phi_{d j}=\delta \phi_{S j}+\omega_{d} \delta t_{S j} \\
\delta \phi_{S j}=\phi-\phi_{S j}, \quad \delta t_{S j}=t-t_{S j}
\end{array}
$$

and $\phi_{S j}$ and $t_{S j}$ are the $\phi$ and $t$ of the satellite at the $j$ th $L$ shell encounter. All $L$-dependent quantities in the integrand of (12) are evaluated at $L^{\prime}$. The solution (12) is a generalization to two dimensions of the one-dimensional calculations of Mead and Hess [1973] and Van Allen et al. [1980].
The first term in (12) represents the effects of radial diffusion on $f_{0}$ (note that if $f_{0}$ were $\phi$ dependent, then azimuthal diffusion would also be included in this term). The second term takes into account the satellite absorption, and we associate it with the microsignature, $f_{I}(L, \phi, t)$. Replacing $f\left(L^{\prime}, \phi_{S j}, t_{S j}\right)$ by $f_{A}(L)$ (this approximation is discussed below) yields

$$
\frac{f_{I}}{f_{A}} \approx-\int d L^{\prime} \sum_{j} \frac{\Delta t_{S j} \omega_{d}}{4 \pi\left(D_{L L} D_{\phi \phi}\right)^{1 / 2} \delta t_{S j}} e^{\frac{-1}{4 \delta t_{S j}}\left[\frac{\left(L-L^{\prime}\right)^{2}}{D_{L L}}+\frac{\delta \phi_{d j}^{2}}{D_{\phi \phi}}\right]}
$$

This solution, evaluated at the time of the observed 1989N1 signature, is illustrated in Figure 9. The microsignature is generally small relative to the average signature except at the recent locations of the satellite where it approximates a $\delta$ function. These locations are shown by the narrow, dark bands sloping down to the right. Note that the width of the band becomes smaller than the spatial grid size on which the calculation was made, so the solution is not spatially resolved near the satellite's present location. Because of the rapid electron drifts and the large energy bandwidths of the CRS detectors, much of the complexity of the solution in Figure 9 will be lost in converting such solutions to observed counting rates. The features formed by the satellite at its minimum orbital $L$ values ( $L \approx 4.5$ and 4.8 ) will be least affected by drifts and have strong signatures in the counting rates because they extend over large ranges in longitude.

Figure 9 may be understood qualitatively by considering the satellite orbit in $L$ versus $\phi$ as shown in Figure 5. If the electrons did not drift, then the satellite would sweep out only the electrons along this trajectory. When drifts are included, then in order to represent the sweeping regions the trajectory becomes stretched in the $\phi$ direction by $\omega_{d} \delta t_{s j}$. Many drifts around the planet can occur in the time $\delta t_{S j}$ since the absorption occurred, so the pattern becomes multivalued. It also becomes distorted by the

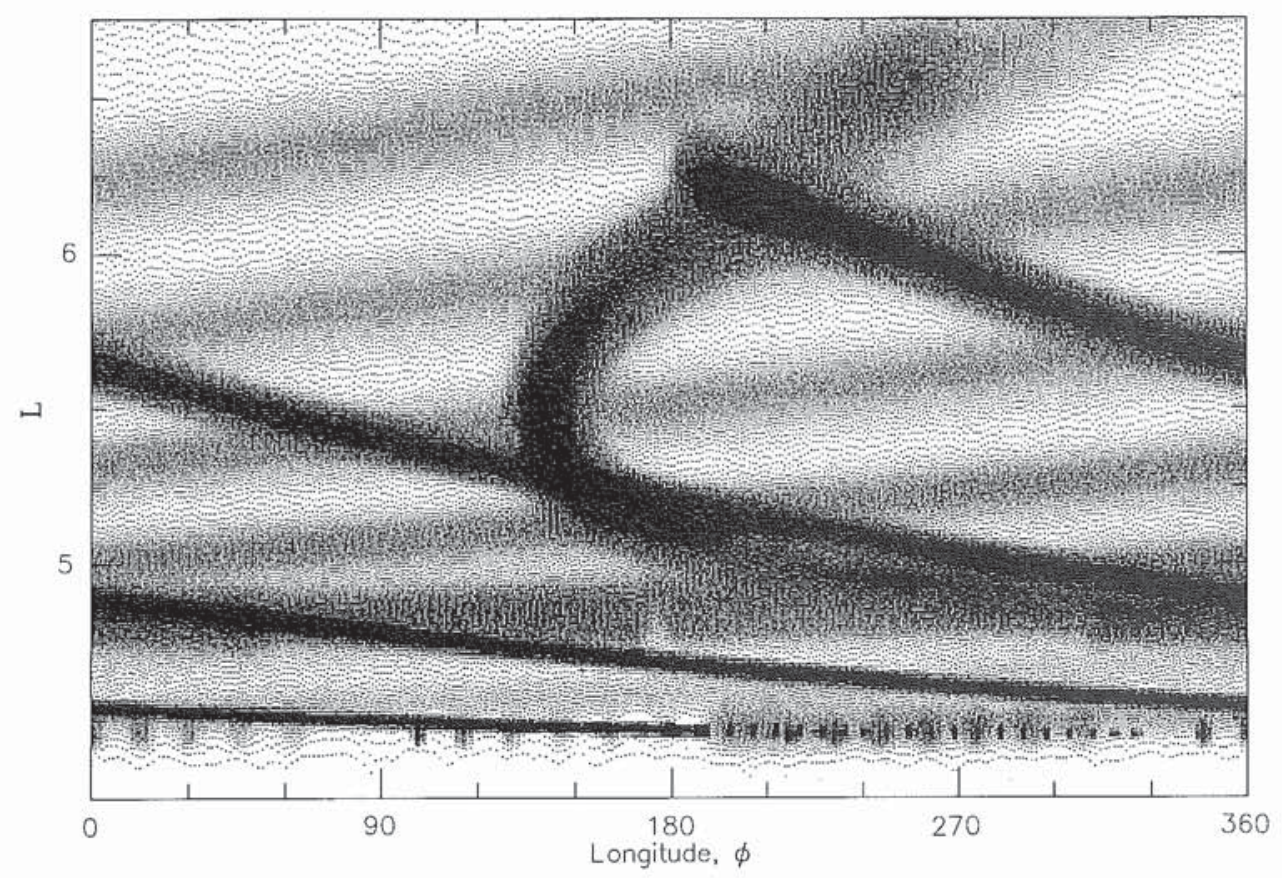

Fig. 9. Grayscale plot of an approximate solution (14) to the diffusion equation at the time ( 0510 SCET) of the observed $1989 \mathrm{~N} 1$ absorption signature, versus $L$ and $\phi$ for fixed values of the first two adiabatic invariants, $M=500 \mathrm{MeV} \mathrm{G}$ and $K=0.3 \mathrm{G}^{1 / 2} \mathbf{R}_{\mathrm{N}}$. The darker shading corresponds to larger absolute value of $f_{I} \mid f_{A}$. The present location of the satellite is $L \sim 4.5, \phi \sim 90^{\circ}$. The diffusion coefficients are as in Figure $8 a$. 
dependence of $\omega_{d}$ on $L$, which causes the C-shaped feature near the center of the plot.

The radial diffusion coefficient, $D_{L L}$, was estimated from the the observed average signature. It may be reasonable to assume, as in Figure 9, that the azimuthal diffusion coefficient, $D_{\phi \phi}$, has a comparable value. However, the energy dispersion effect causes much of the longitude dependence in the microsignature to be lost when averaging over a range of energies, and an assumed value of $D_{\phi \phi}$ is not required to calculate the observed microsignature, as we now demonstrate.

The condition that the hole in the electron population created by absorption drifts from the satellite to the spacecraft is

$$
\delta \phi_{d j}=\delta \phi_{s j}+\omega_{d}\left(E_{j n}\right) \delta t_{s j}=2 \pi n, \quad n=0,1,2,3 \cdots
$$

where $\omega_{d}$ is evaluated at the energy $E_{j n}$ of electrons that drift $n$ times around the planet before encountering the spacecraft. When $n$ becomes large, the energy difference between adjacent $E_{j n}$ (those with $n$ values differing by 1 ) is

$$
\Delta E_{j n}=\frac{2 \pi}{\left(\partial \omega_{d} / \partial E_{j n}\right) \delta t_{S j}}
$$

The CRS counting rates involve integration over energy of the detector response function, $R(E)$, times the electron intensity, $j(E, \alpha, L)$. We assume that $j=k(\alpha, L) E^{-\gamma}$ with $\gamma=6$ (this assumption is consistent with the energy spectra reported by Stone et al. [1989] and with the spectrum obtained as an outer boundary condition for the average signature solution). When the threshold energy of the CRS detector is high enough such that $n$ is large for all energies for which $R(E)>0$, then the integration over energies can be approximated using (16):

$$
\frac{1}{2 \pi} \int \omega_{d} R(E) E^{-\gamma} d E=\sum_{n} \frac{\omega_{d}\left(E_{j n}\right)}{\left(\partial \omega_{d} / \partial E_{j n}\right) \delta t_{S j}} R\left(E_{j n}\right) E_{j n}^{-\gamma}
$$

The microsignature as observed in a CRS detector is obtained by substituting $j=p^{2}\left(f_{A}+f_{l}\right)$ into the counting rate integral (1), with $f_{I}$ from (14) and $p^{2} f_{A}=k E^{-\gamma}$. For simplicity we neglect the pitch angle dependence of $j$ and include only equatorial pitch angles of $\alpha_{0}=30^{\circ}$, which is approximately the value for particles mirroring at the spacecraft when the signatures were observed. We consider two limiting cases. First, for $D_{\phi \phi}=0$ the result is

$$
\begin{aligned}
& \frac{r}{r_{A}}=1-\int d L^{\prime} \sum_{j} \frac{\Delta t_{S j}}{\left(4 \pi D_{L L}\right)^{1 / 2} \delta t_{S_{j}}^{3 / 2}} e^{\frac{-\left(L-L^{\prime}\right)^{2}}{4 D_{L L^{\delta t}} j j}} \\
& \cdot \frac{1}{N} \sum_{n} \frac{\omega_{d}\left(E_{j n}\right)}{\left(\partial \omega_{d} / \partial E_{j n}\right)} R\left(E_{j n}\right) E_{j n}^{-\gamma}
\end{aligned}
$$

where $r_{A}$ is the counting rate for $f=f_{A}$ and the normalization constant $N=\int R(E) E^{-\gamma} d E$. Second, in the case where $D_{\phi \phi}$ approaches infinity, the result is

$$
\begin{array}{r}
\frac{r}{r_{A}}=1-\int d L^{\prime} \sum_{j} \frac{\Delta t_{S j}}{\left(4 \pi D_{L L} \delta t_{S j}\right)^{1 / 2}} e^{\frac{-\left(L-L^{\prime}\right)^{2}}{4 D_{L L} \delta t_{S j}}} \\
\frac{1}{2 \pi N} \int \omega_{d} R(E) E^{-\gamma} d E
\end{array}
$$

The two results (18) and (19) were obtained without the approximation (17), which now shows that they are equal if $R(E)>0$ only for energies with large $n$. Therefore, since it is a monotonic function of $D_{\phi \phi}$, the observed microsignature becomes independent of $D_{\phi \phi}$ as it ages. By (19) it also becomes independent of longitude $\phi$.

In the case where radial diffusion is neglected $\left(D_{L L}=0\right),(18)$ and (19) reduce to

$$
\frac{r}{r_{A}}=1-\frac{1}{N} \sum_{j, n} R\left(E_{j n}\right) E_{j n}^{-\gamma} \delta E_{j n}
$$

and

$$
\frac{r}{r_{A}}=1-\sum_{j} \frac{\Delta t_{S j}}{2 \pi N} \int \omega_{d} R(E) E^{-\gamma} d E
$$

where

$$
\delta E_{j n}=\frac{\Delta t_{S j} \omega_{d}}{\left(\partial \omega_{d} / \partial E_{j n}\right) \delta t_{S j}}
$$

The energy difference $\delta E_{j n}$ is interpreted as the range of energies that are absorbed, in a given episode $j$ and for a given number of drift orbits $n$, because of the finite radial width of the satellite. In fact, under such an interpretation, (22) may be derived directly from the definitions of $E_{j n}$ (condition (15)) in the small satellite limit and of the satellite radial width $\Delta L_{S}$ (equation (7)). From (21), $r / r_{A}$ is seen to become independent of time as well as of $\phi$ and $D_{\phi \phi}$. Thus, for a given detector and with $D_{L L}=0$, the observed microsignature reflects only the $L$ shell contact time $\Delta t_{S j}$.

Simulations of the $1989 \mathrm{~N} 1$ observed microsignatures are illustrated in Figure 10. The simulations were started at a time one orbital period, $T_{S}$, prior to the beginning of the most recent, set of $L$ shell encounters at $\sim 1800$ SCET on day $236, \phi \sim 190^{\circ}$ (see Figure 5). There are therefore up to five absorption episodes at a given $L$. The top panel shows the $E_{j n}$ versus $L$ for the energy range relevant to the D1 counting rate. The number $n$ of drifts around the planet increases with energy and at these energies, except for the most recent episode, is large. The bottom panel shows the simulated normalized counting rates, $r / r_{A}$, for the D1 and D2 detectors in three cases. The case with no diffusion is from (20), the case with radial diffusion but with $D_{\phi \phi}=0$ is from (18), and the case with radial diffusion and strong azimuthal diffusion is from (19). The last case does not include the most recent absorption episodes, which are therefore represented by the difference in normalized counting rate between the two cases with radial diffusion and are little affected by diffusion in the short time since they were formed. The two cases with diffusion are indistinguishable for all absorption episodes prior to the most recent indicating that the large $n$ approximation discussed above is valid for these episodes. Since diffusion is insignificant for the most recent episodes, all of the results are independent of the value of $D_{\phi \phi}$. Therefore the case with $D_{\phi \phi}=0$, shown by the solid curve in the bottom panel, is a good approximation of the microsignatures due to all of the absorption episodes.

The name "microsignature" is sometimes used to describe only those signatures that have drifted less than one time, or a few times, around the planet. We choose to use the name for all of the signatures in Figure 10, including those in the large $n$ limit, because they can lead to small-scale, transient features in the data. Our usage does suffer from the disadvantage of making the distinction between microsignatures and macrosignatures somewhat arbitrary.

The D2 simulation in Figure 10 for the case of no diffusion falls below zero near each of the satellite minimum $L$ shell locations. This unphysical result is due to the replacement of 


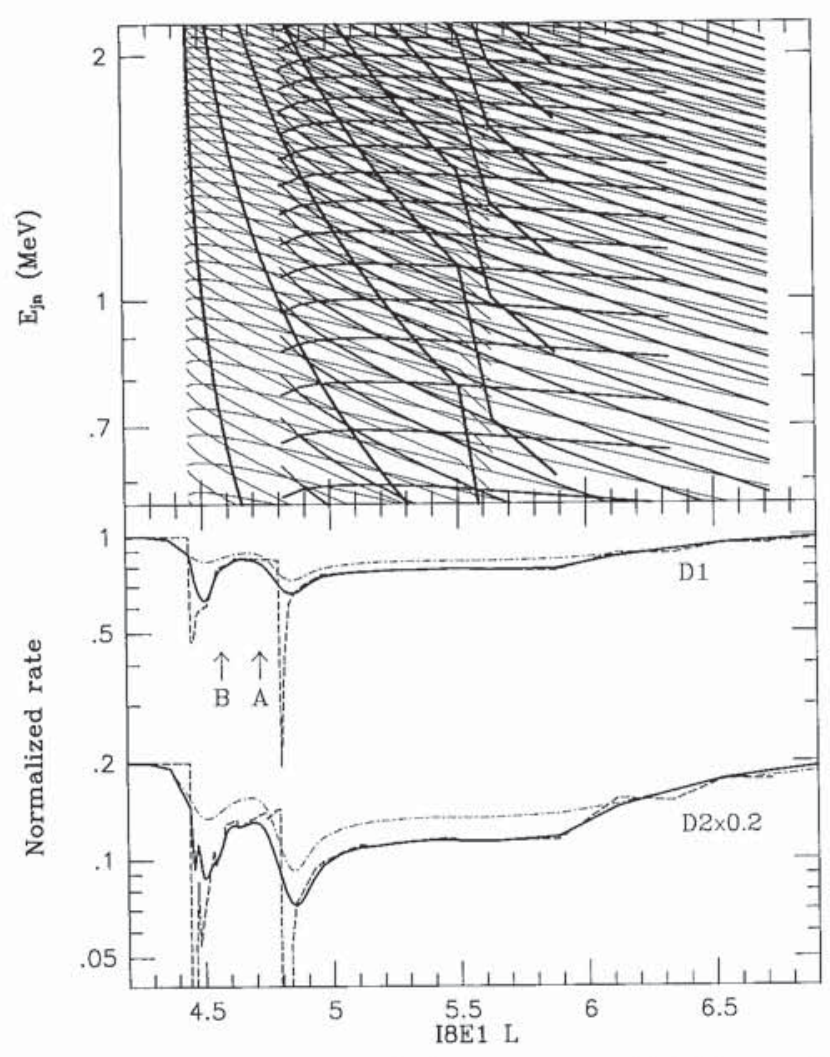

Fig. 10. The top panel shows kinetic energy versus $L$ of electrons that could have drifted from the location of $1989 \mathrm{~N} 1$ to Voyager 2 . The curves increase in weight for more recent encounters of $1989 \mathrm{~N} 1$ with each $L$ shell. The bottom panel shows simulated normalized counting rates due to the microsignatures for the D1 and D2 detectors for three cases: with radial diffusion and no azimuthal diffusion (solid curve), without diffusion (dashed curve), and with radial diffusion and strong azimuthal diffusion (light dash-dot curve). The last case does not include the most recent absorption episodes. The radial diffusion coefficients are equal to that of Figure $8 a$. The A and B labels correspond to those in Figure 2.

$f\left(L^{\prime}, \phi_{S j}, t_{S j}\right)$ in (12) with $f_{A}$ in (14). That is, we have not taken into account overlap of the absorption energy intervals, $\delta E_{j n}$, for different values of $j$ and $n$. The overlaps could have been included, at the cost of computational complexity, by retaining the original solution (12), but this was unnecessary for two reasons. First, the high time resolution data are only available for the D1 detector, and the amount of overlap is insignificant for the D1 simulations. Second, when diffusion is included, the signatures of a given absorption episode substantially fill in before the next episode at the same $L$, so that the $j$ overlaps are less important. The $n$ overlaps are generally insignificant.

Microsignature calculations for the case of no diffusion have been made by Chenette and Stone [1983] for Mimas and by McKibben et al. [1983] for Amalthea. These authors did not make the approximation of neglecting the satellite radius. Their results are therefore more accurate in the case where diffusion is negligible, but for the $1989 \mathrm{~N} 1$ signature the effects of diffusion can be more important than the neglect of the satellite radius, as shown by the substantial filling in of the older signatures in Figure 10. Even in the case of no diffusion, the decrease in the D1 counting rate due to the microsignature is considerably smaller than that due to the average signature. This is because the energy dependence of $\omega_{d}$ causes the microsignatures, which are initially formed at all energies, to disperse onto the discrete set of energies $E_{j n}$ illustrated in the top panel of the figure. The importance of the energy dispersion was also pointed out by the previous authors [Chenette and Stone, 1983; McKibben et al., 1983]. Equations (18) and (19) show that the older microsignatures approach the conditions assumed by Van Allen et al. [1980] of one-dimensional diffusion and no drifts, whereas for the most recently formed microsignatures, diffusion can be neglected and the energy dispersion caused by the drifts must be included.

The approximation of neglecting the $L$ dependence of $\omega_{d}$ in the solution (12) means that dispersion in $L$ is not included for a signature that has spread radially by diffusion (note that the $L$ dependence of $\delta \phi_{d j}$ in (13a) is included to keep proper track of the signature locations). However, since for large $n$ the observed microsignature becomes independent of $\phi$, the $L$ dispersion becomes insignificant, and the approximation is a valid one.

\subsection{The Macrosignature Approximation}

The macrosignature is the large-scale signature of satellite absorption. Therefore it includes both the average signature, $f_{A}$, and any part of the local signature that would be observed as a large-scale feature relative to the microsignature. The first term of the solution (12), which involves the initial condition $f_{0}$, should therefore be included in the macrosignature. If a complete solution of the full diffusion equation (8) were allowed to evolve for many satellite orbits, then it would become independent of $f_{0}$, and the distinction between the macrosignature and the microsignature would not be necessary. Since we have not used such a solution, we must consider the accuracy of the approximations we have made.

The approximation made in deriving the average diffusion equation (10) was

$$
\tau_{S}^{-1} f\left(L, \phi_{S j}, t_{S j}\right) \approx \tau_{S}^{-1} f_{A}(L)
$$

which is valid if the $t$ and $\phi$ dependencies of $f$ are small. That is,

$$
\frac{T_{S}}{f_{A}} \frac{\partial f_{L}}{\partial t} \ll 1 \quad \text { and } \quad \frac{2 \pi}{f_{A}} \frac{\partial f_{L}}{\partial \phi} \ll 1
$$

which are also the conditions that the average signature be the dominant part of $f$. They are required only away from the satellite where the loss term in the diffusion equation (8) is zero. Substituting the average diffusion equation (10) into (8) without the loss term and using the definition $(9 b)$ of $f_{L}$ yields

$$
\frac{\partial f_{L}}{\partial t}+\omega_{d} \frac{\partial f_{L}}{\partial \phi}=L^{2} \frac{\partial}{\partial L}\left[\frac{D_{L L}}{L^{2}} \frac{\partial f_{L}}{\partial L}\right]+D_{\phi} \frac{\partial^{2} f_{L}}{\partial \phi^{2}}+\left\langle\tau_{s}^{-1}\right\rangle f_{A}
$$

If the diffusion terms were large compared to the average loss term, then $f_{L}$ would quickly fill in by diffusion, and $f_{A}$ would not show a deep signature of the satellite. With neglect of the diffusion terms and substitution of the definition (11) of $\left\langle\tau_{s}^{-1}\right\rangle$, the conditions (24) require

$$
\frac{\Delta t_{S} \omega_{d}}{2 \pi} \ll 1 \quad \text { and } \quad \frac{\Delta t_{S}}{T_{S}} \ll 1
$$

For 1989N1 the second condition in (26) is always satisfied because the maximum $L$ shell contact time $\Delta t_{S}$ is $\sim 1$ hour (Figure 6 ) and the orbital period $T_{S}$ is $\approx 40$ hours. The first condition is not strictly satisfied because the drift period for $\sim 1$ $\mathrm{MeV}$ electrons at $L=5$ is $2 \pi / \omega_{d} \sim 2$ hours.

The violation of the first condition in (24) and (26) says that the fill-in of the macrosignature between sweeping episodes may not be negligible. The fill-in during one orbital period of $1989 \mathrm{~N} 1$ is 


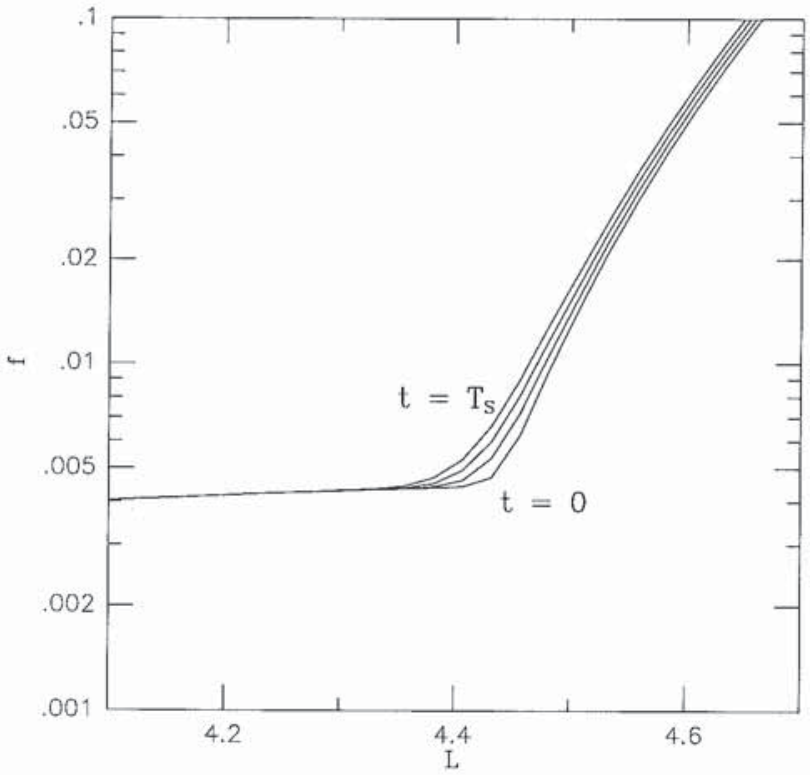

Fig. 11. The time-dependent filling in of the macrosignature during one orbital period $T_{S}$ of $1989 \mathrm{~N} 1$. Time increases by equal increments from the lower curve to the upper curve.

illustrated in Figure 11 using the first term of the time-dependent solution (12). Although the macrosignature does fill in substantially at the inner orbital minimum $L$, the change in $f$ by a factor $\sim 1.5$ is considerably smaller than the factor $\sim 20$ decrease in the counting rate observed upon entering the satellite signature. Therefore the observed signature can be interpreted as being substantially the steady state average signature, with the remainder including small contributions from both the microsignature and the slow fill-in. These conclusions are valid over a large range of energies because although the fill-in is faster at higher energies, the average signature is proportionately deeper. Since the contribution from the fill-in is small, we can now refer to the average signature, $f_{A}$, as the macrosignature.

Finally, the derivation of the average lifetime (11) included a similar approximation to that discussed above with regard to the microsignature calculations in neglecting the overlap in energy of different absorption episodes. Therefore the average lifetime at energies relevant to the D2 detector can be too large, depending on the value of the diffusion coefficient. Again, the full solution (12) must be used to evaluate this effect. It should not be large for the value of $D_{L L}$ we have used because of the significant filling in of the microsignatures between episodes.

\section{DISCUSSION}

The macrosignature simulations of Figure 8 showed that the depth of the counting rate signature is sensitive to the value of the radial diffusion coefficient, $D_{L L}$. Also the shape of the observed signature is sensitive to the radial dependence of $D_{L L}$. Therefore, the observed depth and shape provide strong constraints on $D_{L L}$, the derived value of which is then reliable to the extent that the model is valid. That is, if radial diffusion due to violation of the third adiabatic invariant is the dominant mechanism for replenishing losses and if absorption by $1989 \mathrm{~N} 1$ is the dominant loss mechanism, then $D_{L L} \approx 2 \times 10^{-8}(L / 5)^{7} \mathrm{~s}^{-1}$. The uncertainty in this value within the model is equivalent to the amount of filling in of the macrosignature between sweeping episodes, which we have shown to be a factor of $\sim 1.5$. The value is in reasonable agreement with estimates of $D_{L L}$ for the magnetosphere of Uranus [Hood, 1989b]. However, the radial dependence is inconsistent with an $L^{3}$ dependence which has been inferred from satellite signatures at Jupiter [Thomsen et al., 1977a], Saturn [Hood, $1989 a$ ], and Uranus [Hood, 1989b] and interpreted as evidence for diffusion driven by an atmospheric dynamo. Therefore diffusion at Neptune may be driven by external factors which lead to a steeper radial dependence [e.g., Schulz and Lanzerotti, 1974]. However, the model is not as sensitive to the radial dependence of $D_{L L}$ as it is to the value at $L \sim 5$. The calculations for the other planets were based on data covering larger radial ranges than those in the present study, and on the signatures of more than one satellite. Therefore the results may not be directly comparable. Also, Hood [1985] has shown that the inclusion of pitch angle scattering losses can reduce the inferred radial dependence of $D_{L L}$.

The principal uncertainty in the macrosignature model is probably the assumption that diffusion occurs only by third invariant violation. At Uranus there is evidence for significant pitch angle and energy diffusion which violate all three adiabatic invariants and lead to an apparent local source of electrons at fixed $M$ and $K$ in the CRS energy range [Selesnick and Stone, 1991]. However, the success of the model in matching the 1989 N1 signature suggests that such processes are not as significant at Neptune, a result that may be consistent with the lower level of plasma wave activity in Neptune's magnetosphere compared with that of Uranus [Gurnett et al., 1989]. The relative depths of the D1 and D2 model signatures show some discrepancies from the data, due to the softening of the model electron energy spectra in the absorption region (Figure 7). This may indicate that energy diffusion is required in the model. Future work in modeling a larger radial extent of Neptune's radiation belt will further address these issues.

Although the macrosignature model was reasonably successful in accounting for the approximate shape and depth of the observed signature, the predicted location was inside that observed by $\sim 0.3$ unit in $L$. A similar displacement was observed in the high-latitude Ariel signature at Uranus [Selesnick and Stone, 1991]. However, the interpretation of that displacement depended on the concurrent observation of an apparent local electron source, associated with a local minimum in $f$ that was not required in modeling the $1989 \mathrm{~N} 1$ signature. Also, the Ariel displacement was seen to increase with energy [Cooper and Stone, 1991], so that it could not have been a result of uncertainties in the magnetic coordinates. When comparing the CRS data with electron observations at 252 to $480 \mathrm{keV}$ [Krimigis et al., 1989], such an energy dependence is not apparent in the $1989 \mathrm{~N} 1$ signature. An alternative explanation for the $1989 \mathrm{~N} 1$ displacement may be uncertainties in the actual minimum $L$ shell values of the orbit. The absolute minimum of the observed counting rate signature is at $L \approx 4.72$, near the orbital distance of $1989 \mathrm{~N} 1\left(4.75 R_{N}\right)$. The displacement of the predicted signature is a result of the radial separation of the two orbital minimum $L$ values around this value, by $\sim 0.34$ unit in $L$ according to the I8E1 magnetic field model. Further work is required to evaluate the accuracy of the separation distance in the field model.

The counting rate minima in the microsignature simulations of Figure 10 , in the case when diffusion is included, have a small outward displacement relative to those in the case with no diffusion, due to the convolution with a Gaussian of the asymmetrical drift shell contact time. The displacement $(\sim 0.05$ unit in $L$ ) is small compared with that of the observed counting rate minima from the predictions of the macrosignature simulations.

The results of Figure 10 show that without diffusion the predicted microsignatures would have sharp local minima at the 
two orbital minimum $L$ values of 1989 N1. In fact, the high time resolution data in Figure 2 show two sharp local minima in the D1 counting rate, one at the deep minimum associated with the macrosignature (labeled $\mathrm{A}$ in Figure 2), and one inside by 0.15 units in $L$ (labeled $\mathrm{B}$ ). Unfortunately the inner counting rate minimum could not be seen in its entirety owing to the instrumental mode change at that time. It may not be as sharp as it appears if the number of counts in the first point of the high resolution data was too low because of a $2 \sigma-3 \sigma$ fluctuation. Then the true inner minimum could be somewhat inside its apparent location. However, it appears likely that the actual location was near that shown on the figure. The locations in $L$ shell corresponding to the $\mathrm{A}$ and $\mathrm{B}$ labels are indicated on Figure 10.

The two local counting rate minima may represent the true locations of the orbital minimum $L$ values of $1989 \mathrm{~N} 1$, after correcting for the small outward displacements suggested by the microsignature calculations in Figure 10. If so, then Figure 10 shows that the minimum $L$ values are somewhat closer together than predicted by the I8E1 magnetic field model. Since the absolute counting rate minimum is observed to be at the outer local minimum, rather than at the inner local minimum as predicted by the diffusion model, the macrosignature would have to be displaced outward from its expected location as in the Ariel signature at Uranus. The outer local minimum (A in Figure 2) appears to be somewhat sharper than predicted in Figure 10 where it has been broadened by diffusion. Therefore the estimated radial diffusion coefficient may be too high. Alternatively, the outer signature may be at the location of the inner orbital minimum $L$, in which case the macrosignature would not have to be displaced from its expected location. Then a new explanation would be required for the inner counting rate minimum ( $B$ in Figure 2). One possibility is a recently formed microsignature of orbital material that was near the $1989 \mathrm{~N} 1$ minimum $L$ shells. At least one of the observed microsignatures must be due to recent absorption by $1989 \mathrm{~N} 1$ at its inner minimum orbital $L$ because that microsignature could not yet have filled in by diffusion. Modeling electron absorption by material orbiting near or inside the orbit of $1989 \mathrm{~N} 1$, or establishing a mechanism for displacing the macrosignature outwards would help to distinguish between the possibilities of one or both of the observed microsignatures being due to $1989 \mathrm{~N} 1$.

Acknowledgments. We thank N. F. Ness, J. E. P. Connerney, and M. H. Acuna for providing the magnetic field model parameters. This work was supported by NASA under contract NAS7-918 and grants NAGW-1919 and NAGW-2402.

The editor thanks L. L. Hood and D. L. Chenette for their assistance in evaluating this paper.

\section{REFERENCES}

Birmingham, T. J., T. G. Northrop, and C.-G. Falthammer, Charged particle diffusion by violation of the third adiabatic invariant, Phys. Fluids, 10, 2389-2398, 1967.

Chenette, D. L. and L. Davis, Jr., An analysis of the structure of Saturn's magnetic field using charged particle absorption signatures, J. Geophys. Res., 87, 5267-5274, 1982.

Chenette, D. L. and E. C. Stone, The Mimas ghost revisited: An analysis of the electron flux and electron microsignatures observed in the vicinity of Mimas at Saturn, J. Geophys. Res., 88, 8755-8764, 1983.

Connerney, J. E. P., M. H. Acuna, and N. F. Ness, The magnetic field of Neptune, J. Geophys. Res., this issue.

Cooper, J. F., Satellite sweeping of electrons at Neptune and Uranus, Geophys. Res. Lett., 17, 1665-1668, 1990.

Cooper, J. F. and E. C. Stone, Electron signatures of satellite sweeping in the magnetosphere of Uranus, J. Geophys. Res., 96, 7803-7821, 1991.

Cuzzi, J. N. and J. A. Burns, Charged particle depletion surrounding Saturn's F ring: Evidence for a moonlet belt?, Icarus, 74, 284-324, 1988.
Fillius, W., Toward a comprehensive theory for the sweeping of trapped radiation by inert orbiting matter, J. Geophys. Res., 93, 14,284-14,294, 1988.

Gurnett, D. A., W. S. Kurth, R. L. Poynter, L. J. Granroth, I. H. Cairns, W. M. Macek, S. L. Moses, F. V. Coroniti, C. F. Kennel, and D. D. Barbosa, Plasma wave observations at Neptune, Science, 246, 1494-1498, 1989.

Hood, L. L., Radial diffusion of low-energy ions in Satum's radiation belts: A combined analysis of phase space density and satellite microsignature data, J. Geophys. Res., 90, 6295-6303, 1985.

Hood, L. L., Radial diffusion and losses of energetic protons in the 5 to 12 $R_{S}$ region of Saturn's magnetosphere, J. Geophys. Res., 94, 8721-8730, $1989 a$.

Hood, L. L., Radial diffusion in the Uranian radiation belts: Inferences from satellite absorption loss models, J. Geophys. Res., 94, $15,077-15,088,1989 b$.

Krimigis, S. M., T. P. Armstrong, W. I. Axford, C. O. Bostrom, A. F. Cheng, G. Gloeckler, D. C. Hamilton, E. P. Keath, L. J. Lanzerotti, B. H. Mauk, and J. A. Van Allen, Hot plasma and energetic particles in Neptune's magnetosphere, Science, 246, 1483-1489, 1989.

McIlwain, C. E., Magnetic coordinates, Space Sci. Rev., 5, 585-598, 1966.

McKibben, R. B., K. R. Pyle, and J. A. Simpson, Pioneer 11 observations of trapped particle absorption by Amslthea, J. Geophys. Res., 88, $36-44,1983$

Mead, G. P. and W. N. Hess, Jupiter's radiation belts and the sweeping effect of its satellites, J. Geophys. Res., 78, 2793-2811, 1973.

Ness, N. F., M. H. Acuna, L. F. Burlaga, J. E. P. Connerney, R. P. Lepping, and F. M. Neubauer, Magnetic fields at Neptune, Science, 246, 1473-1478, 1989.

Paonessa, M. T. and A. F. Cheng, Satellite sweeping in offset, tilted dipole fields, J. Geophys. Res., 92, 1166, 1987.

Press, W. H., B. P. Flannery, S. A. Teukolsky, and W. T. Vetterling, Numerical Recipes: The Art of Scientific Computing, Cambridge University Press, New York, 1986.

Roederer, J. G., Dynamics of Geomagnetically Trapped Radiation, Springer, New York, 1970.

Schulz, M. and L. J. Lanzerotti, Particle Diffusion in the Radiation Belts, Springer, New York, 1974.

Selesnick, R. S. and E. C. Stone, Energetic electrons at Uranus: Bimodal diffusion in a satellite limited radiation belt, J. Geophys. Res., 96, $5651-5665,1991$.

Simpson, J. A., T. S. Bastian, D. L. Chenette, R. B. McKibben, and K. R. Pyle, The trapped radiations of Satum and their absorption by satellites and rings, J. Geophys. Res., 85, 5731-5762, 1980.

Stone, E. C., The physical significance and application of $L, B_{0}$, and $R_{0}$ to geomagnetically trapped particles, J. Geophys. Res., 68, 4157-4166, 1963.

Stone, E. C., J. F. Cooper, A. C. Cummings, F. B. McDonald, J. H. Trainer, N. Lal, R. McGuire, and D. L. Chenette, Energetic charged particles in the Uranian magnetosphere, Science, 233, 93-97, 1986.

Stone, E. C., A. C. Cummings, M. D. Looper, R. S. Selesnick, N. Lal, F. B. McDonald, J. H. Trainor, and D. L. Chenette, Energetic charged particles in the magnetosphere of Neptune, Science, 246, 1489-1494, 1989.

Stone, E. C., R. E. Vogt, F. B. McDonald, B. J. Teegarden, J. H. Trainer, J. R. Jokipii, and W. R. Webber, Cosmic ray investigation for the Voyager missions: Energetic particle studies in the outer heliosphere - and beyond, Space Sci. Rev., 21, 355-376, 1977.

Thomsen, M. F., Jovian magnetosphere-satellite interactions: Aspects of energetic charged particle loss, Rev. Geophys., 17, 369-387, 1979.

Thomsen, M. F., C. K. Goertz, and J. A. Van Allen, A determination of the $L$ dependence of the radial diffusion coefficient for protons in Jupiter's inner magnetosphere, J. Geophys. Res., 82, 3655-3658, $1977 a$.

Thomsen, M. F., C. K. Goertz, and J. A. Van Allen, On determining magnetospheric diffusion coefficients from the observed effects of Jupiter's satellite Io, J. Geophys. Res., 82, 5541-5550, 1977 b.

VanAllen, J. A., M. F. Thomsen, and B. A. Randall, The energetic charged particle absorption signature of Mimas, J. Geophys. Res. , 85, 5709-5718, 1980.

R. S. Selesnick and E. C. Stone, 220-47 California Institute of Technology, Pasadena, CA 91125.

(Received February 4, 1991; revised June 13, 1991; accepted June 14, 1991.) 
